DOI: 10.18605/2175-7275/cereus.v10n2p240-251

<<Recebido: 04/06/2018. Aceito: 06/07/2018〉>
ARTIGO ORIGINAL

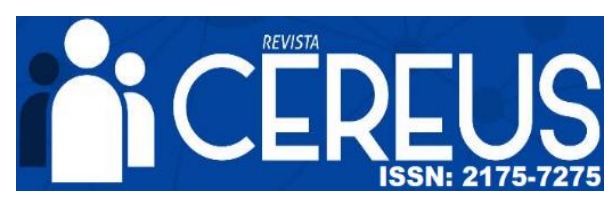

\title{
Processamento Digital de Imagens Aplicado a Identificação Automática de Placas
}

de Trânsito

\section{Digital image processing applied to the automatic identification of traffic signs}

Thiago de Jesus Oliveira Duraes ${ }^{1}$, Lucas Soares Maciel ${ }^{2}$, Wagner Ferreira de Barros $^{3}$

\section{RESUMO}

O estudo da detecção de placas de trânsito tem sido de grande interesse e muitas vezes é abordado por um procedimento em três etapas envolvendo detecção, rastreamento e classificação. O trabalho proposto apresenta um sistema relativamente simples para detecção de placas de sinalização de trânsito, utilizando a placa de parada obrigatória como exemplo. O algoritmo desenvolvido faz uso de um processo de segmentação e detecção de retas para obter as coordenadas das retas nas bordas de objetos vermelhos em uma imagem de entrada possibilitando a detecção de uma possível placa de pare. Para validação do método foram realizados testes em um banco de dados de imagens, onde metade das imagens não contêm placas e, com isto, foram avaliados o número médio de acertos, falsos positivos e falsos negativos obtidos.

Palavras-chave: Detecção de bordas, Segmentação, Identificação, Placas de trânsito, Detecção de retas.

\section{ABSTRACT}

The study of traffic sign detection has been of great interests and often addressed by a three-stage procedure involving detection, tracking and classification. This work presents a relatively simple system for detecting traffic signs, using the stop warning sign as an example. The developed algorithm makes use of a color segmentation algorithm and an efficient method for line detection to obtain the coordinates of the lines at the edges of the red objects presents in an input image, allowing the detection of a possible stop plate. The validation of the method was performed by the average number of hits, false positives and false negatives applied to an image database, where half of the images did not contain traffic signs

Keywords: Edge detection, Segmentation, Identification, Traffic signs, line detection.
${ }^{1}$ Acadêmico do curso Ciência da Computação do IFNMG Campus Montes Claros.

E-mail:

thiagooduraes@gmail.com

${ }^{2}$ Acadêmico do curso Ciência da Computação do IFNMG Campus Montes Claros.

${ }^{3}$ Docente do curso Ciência da Computação do IFNMG Campus Montes Claros. 


\section{INTRODUÇÄO}

Placas de sinalização de trânsito são dispositivos visuais de segurança que tem como objetivo informar aos condutores e pedestres das condições e normas de circulação das vias de transito. São projetadas de forma a ter uma compreensão simples e rápida, para que qualquer pessoa, condutor ou pedestre, possa entender a informação até mesmo em um país estrangeiro.

Com a evolução da tecnologia para automóveis e a popularização de aplicativos de GPS, surge a necessidade de transmitir as informações das placas de trânsito para os sistemas embarcados dos veículos e aplicativos para motoristas.

Segundo LOY AND BARNES (2004), a pesquisa na área de reconhecimento de placas de trânsito é feita desde os anos 1980. A maior parte dos trabalhos utiliza segmentação por cor, geralmente usando os espaços de cores HSV (do inglês: Hue, Saturation and Value) e HSI (Hue, Saturation and Intensity). Estes trabalhos também utilizam o reconhecimento de formas após a segmentação por cor, como o trabalho proposto por ZHANG (2016), que faz um melhoramento da imagem antes da segmentação e detecta as bordas da imagem resultante, e LOY AND BARNES (2004) que procura as formas básicas das placas na imagem segmentada utilizando a simetria das formas e a orientação das arestas presentes nas placas.

Também são feitos trabalhos utilizando apenas o reconhecimento de formas, como LI et al. (2015), que utiliza a detecção de segmentos de reta na imagem por completo e procura por segmentos que formem um octógono. Bem como aqueles que identificam pontos homólogos entre a imagem de entrada e as imagens das placas salvas anteriormente, como o algoritmo SIFT, utilizado por da SILVA et al. (2012), que procura correspondências entre imagens de placas de transito e imagens obtidas por um sistema de mapeamento móvel.

O trabalho proposto apresenta um sistema relativamente simples para detecção de placas de sinalização de trânsito, utilizando a placa de pare como exemplo. Assim, foram utilizadas técnicas convencionais de processamento digital de imagens como métodos de segmentação, detecção de bordas e detecção de segmentos de retas. Para isto, foi utilizado o toolbox de processamento de imagens do Matlab (MATHWORKS, 2017), e seus algoritmos para detecção de bordas como o filtro Sobel e o algoritmo de Canny, operadores morfológicos, para auxiliar no processo de segmentação da placa e, para detecção dos segmentos de retas, foi utilizada a Transformada de Hough. Para validação 
do método foram realizados testes em um banco de dados de imagens, onde metade das imagens não contém placas e, com isto, foram avaliados o número médio de acertos, falsos positivos e falsos negativos obtidos.

\section{METODOLOGIA}

A metodologia proposta para desenvolvimento deste trabalho pode ser dividida nos seguintes passos:

1. Segmentar a imagem de entrada com o objetivo de ressaltar uma porção desta imagem que se encontre na mesma faixa de cores da placa a ser localizada.

2. Processar o objeto identificado para verificar se ele pode ser caracterizado como uma placa de pare.

Para realização do primeiro passo, primeiro é necessário converter a imagem de entrada para um espaço de cores mais adequado e, posteriormente, analisar os histogramas que caracterizam as cores da imagem para extrair da imagem a informação desejada.

De posse do resultado obtido no passo inicial, deve-se agora processar a informação obtida para identificar a forma geométrica do objeto identificado. Para isto, optou-se por identificar os diversos segmentos de retas que contornam o objeto identificado por meio da Transformada de Hough.

\subsection{Espaço de cores HSV}

Para efetuar uma separação de objetos em vermelho na imagem do restante da cena, o algoritmo utiliza o espaço de cores HSV, que foi proposto por Alvy Ray Smith em 1978, (SMITH, 1978), e decompõe o espaço de cores em três bandas: matiz, saturação e brilho (ou valor).

Segundo SMITH (1978), a matiz e a dimensão que define a cor, como vermelho, amarelo, azul. A saturação define a pureza desta cor, quanto mais impura, mais próxima do cinza. A banda valor armazena o brilho da cor, definindo se está mais próximo do branco, maior brilho, ou do preto, menor brilho. Uma descrição visual deste espaço de cor pode ser observada na Fig. 1. 


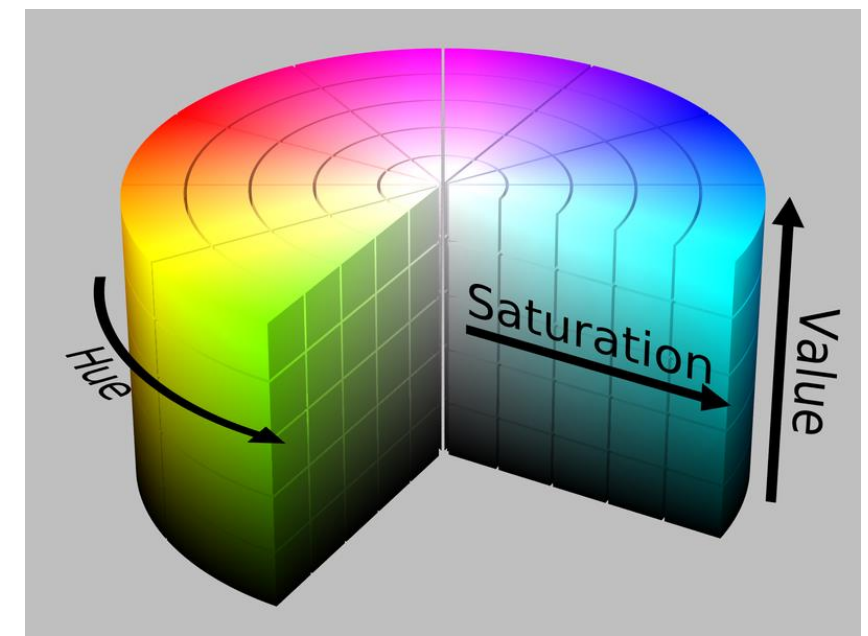

Figura 1. Descrição visual do espaço de cores HSV. Imagem obtida em https://commons.wikimedia.org/w/index.php?curid=9801673 , último acesso em 14/08/2017.

\subsection{TRANSFORMADA DE HOUGH}

Suponha que, dado $n$ pontos em uma imagem deseja-se encontrar a equação de uma reta que passa por estes pontos. A realização de tal tarefa pode ser feita ao encontrar todas as retas determinadas por cada par de pontos e, em seguida, deve-se encontrar todos os subconjuntos de pontos que estejam próximos a essas retas em particular. Tal tarefa teria um custo computacional muito alto (algo em torno de $n^{3}$ comparações) o que a tornaria proibitiva para grande parte das aplicações computacionais GONZALEZ AND WOODS (2014). Em 1962, Hough propôs um método mais eficiente, chamado de transformada de Hough (HT - Hough Transform), HOUGH (1962). A ideia básica é transformar um problema difícil de detecção de curvas em um problema simples de detecção de máximos (ou picos) em um espaço de parâmetros desta curva.

Uma linha reta é descrita como $y=a x+b$. As características desta reta são sua inclinação $\theta=\arctan a$ e a intersecção $b$ com o eixo vertical do sistema de coordenadas. Assim, uma reta $y=a x+b$ pode ser representada como um ponto $(a, b)$ em seu espaço de parâmetros. Por outro lado, esta representação apresenta um inconveniente para representação de retas verticais, uma vez que, neste caso, o parâmetro $a=\tan \theta \rightarrow \infty$. Uma maneira de contornar este problema é utilizar a representação da reta em coordenadas polares no espaço $(\rho, \theta)$ :

$x \cos \theta+y \sin \theta=\rho$.

Segundo KIM (2017), isto associa cada reta da imagem a um único ponto $(\rho, \theta)$ no 
plano dos parâmetros (ou espaço de Hough).

Infinitas retas passam por um ponto no plano. Todas as retas que passam por esse ponto formam um senoide no plano de Hough, como pode ser visto nas Figs. 2(a) e (b).

Dois pontos $p$ e $q$ no plano da imagem definem uma reta $p q$. Dois pontos $p$ e $q$ correspondem a dois senoides no plano de Hough. A intersecção dos dois senoides representa a reta $p q$ que passa pelos dois pontos $p$ e $q$ no plano da imagem (Figs. 2(c) e (d)). Uma reta no plano da imagem corresponde a infinitos senoides no plano de Hough que intersectam num único ponto (Figs. 2(e) e (f)). Este ponto do plano de Hough representa a reta.

Se por outro lado, têm-se 4 retas no plano da imagem, estas correspondem a 4 senoides no plano de Hough que se acumulam em 4 pontos (Figs. 2(g) e (h)). Note que os 2 pontos de acumulação nas bordas esquerda e direita da Figs. 2(h) correspondem a uma única reta, que pode ser representada por $\theta=0$ ou $\theta=\pi$.

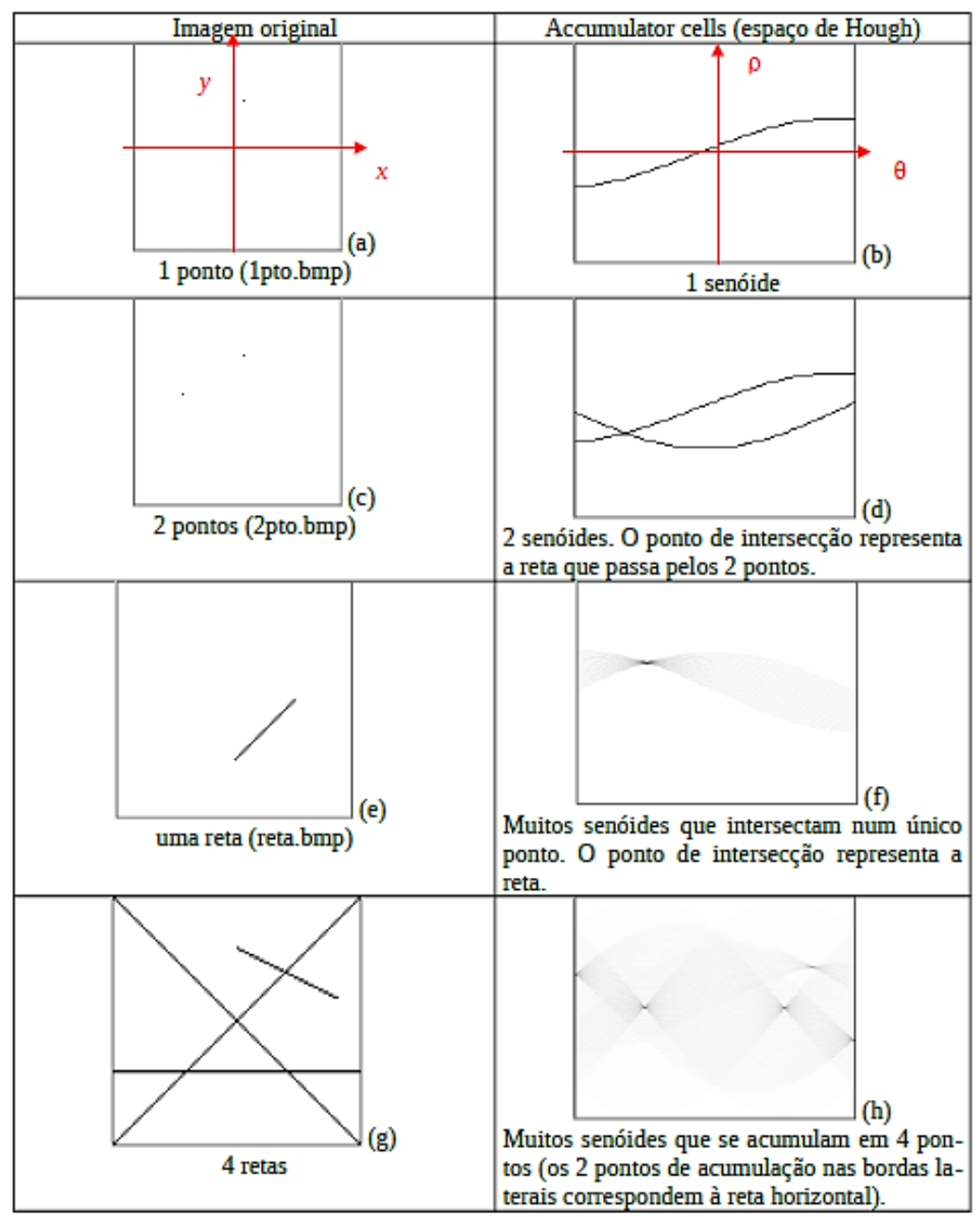

Figura 2. Correspondência entre uma reta no espaço $x y$ e no espaço de Hough. Imagem obtida de Kim (2017). 


\subsection{DESENVOLVIMENTO DO SISTEMA}

Conforme descrito, a metodologia proposta consiste em segmentar uma imagem de uma possível placa de pare, detectar as bordas desta imagem segmentada para aplicar a função da transformada de Hough e detectar as coordenadas das retas presentes nas bordas do objeto detectado para, posteriormente, apresentar as retas detectadas na imagem de entrada.

Todo o trabalho foi implementado utilizando-se o Toolbox de Processamento de Imagens do Matlab, MATHWORKS (2017), no Matlab R2015.

Ao início do algoritmo, é lida a imagem de entrada (no espaço de cores padrão RGB), em que se quer verificar a existência de uma placa de parada obrigatória. A leitura dessa imagem é realizada utilizando a função imread.

Para a detecção de uma placa de pare, primeiramente é feita a separação dos objetos em vermelho do restante da imagem. Esta tarefa é realizada convertendo a imagem para o espaço de cores HSV (função rgb2hsv). Posteriormente, busca-se por pixels com valores de matiz (hue) $10 \%$ mais próximos às extremidades iniciais e finais do conjunto possível de valores representados nesta banda (a matiz armazena as variações cromáticas da imagem de entrada com valores compreendidos entre 0 e 1) e com um valor de saturação acima de 50\% (pois procura-se por cores mais puras). Isto gera uma máscara que é aplicada à imagem original separando os objetos com cor predominantemente vermelha dos demais objetos presentes na imagem. Para finalizar, somente são considerados objetos com dimensão de, no mínimo, 10\% do tamanho total da imagem. Qualquer objeto detectado, com dimensão inferior a $10 \%$ da dimensão da imagem de entrada é descartado pela chamada da função bwareaopen.

Após segmentar um objeto vermelho da imagem, onde uma possível placa de pare estará, a imagem passa por um processo de detecção de bordas pela máscara de gradiente binário nesta imagem segmentada, utilizando o filtro de Sobel aplicado com o auxílio de funções do software.

Depois da aplicação do filtro de Sobel, é possível que bordas do mesmo objeto fiquem desconectadas. Então a imagem gerada é dilatada para realizar a junção de bordas de um mesmo objeto. Essa dilatação é feita através da função imdilate do Matlab.

Como o propósito do algoritmo é detectar apenas as bordas externas de uma possível placa, ao resultado do processo anterior, é aplicado outro operador morfológico para preencher o interior de objetos conectados (função imfill), resultando em uma 
imagem onde, todos os objetos com bordas externas conectadas estão totalmente preenchidos.

$\mathrm{Na}$ metodologia proposta, espera-se que a placa esteja localizada integralmente na área da imagem, dessa forma, é aplicada uma função capaz de remover qualquer objeto conectado que esteja em contato com qualquer extremidade da imagem de entrada (função imclearborder).

Posteriormente, para melhorar a definição geométrica do objeto detectados na imagem, é realizada uma erosão com o auxílio da função imerode, cujo resultado é uma imagem mais bem definida do objeto de interesse.

Neste ponto, espera-se ter uma imagem binária com a geometria bem definida do objeto de interesse (um objeto relativamente grande, na cor vermelha, que não esteja em contato com qualquer extremidade da imagem). Esta imagem é utilizada como entrada no algoritmo de detecção de retas pela transformada de Hough. Neste algoritmo, o primeiro passo é detectar as bordas do objeto de entrada e, para isto, é utilizado o detector de bordas ótimo de Canny. Uma imagem com apenas essas bordas é importante para evitar a detecção de linhas no interior do objeto nos passos a seguir. Fig. 2.3 ilustra o processo.

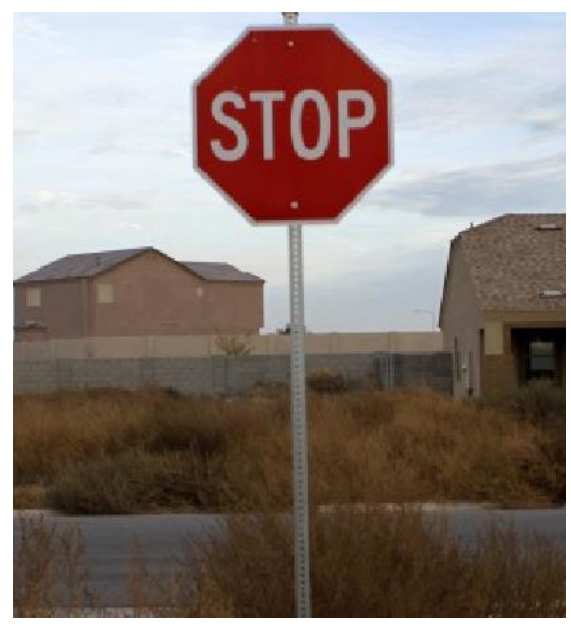

(a)

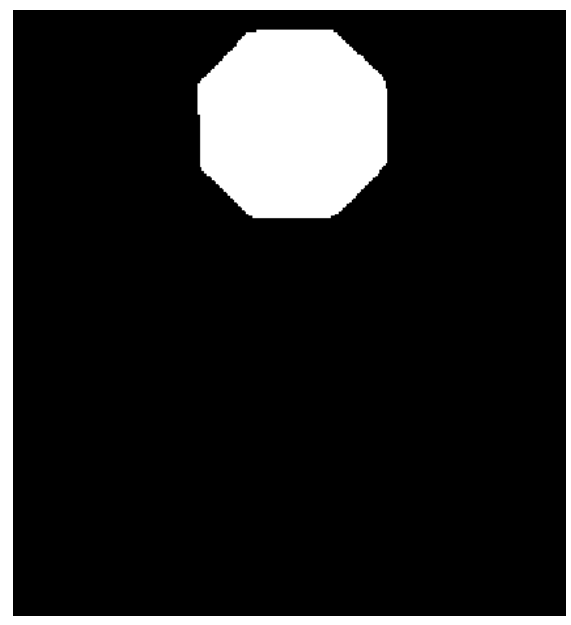

(b)

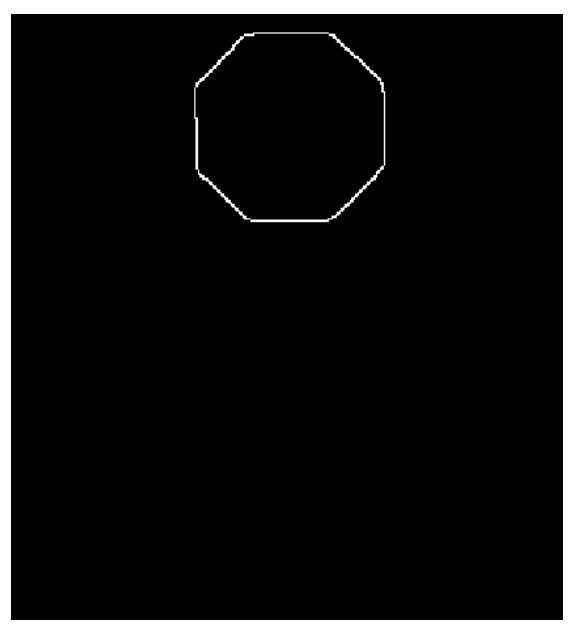

(c)

Figura 3. Passos principais do processo de segmentação da placa. (a) Imagem de entrada. (b) Máscara obtida no processo de segmentação por cores. (c) Bordas obtidas após processamento da máscara.

Com o objeto segmentado e suas bordas detectadas, é aplicada a transformada de Hough para encontrar as retas principais presentes na imagem da placa. A Fig. 4 apresenta o gráfico dos senoides plotados no espaço de Hough para as bordas exibidas na Fig. 3(c). 
A partir do resultado da transformada de Hough, são selecionados, no máximo, oito linhas na imagem, o que é suficiente para detectar uma placa de pare. As linhas detectadas com distância menor ou igual a cinco pixels são unidas formando uma única linha e os segmentos com tamanho menor que sete pixels são descartados. Essas linhas são destacadas no espaço de parâmetros da transformada de Hough, Fig. 4.

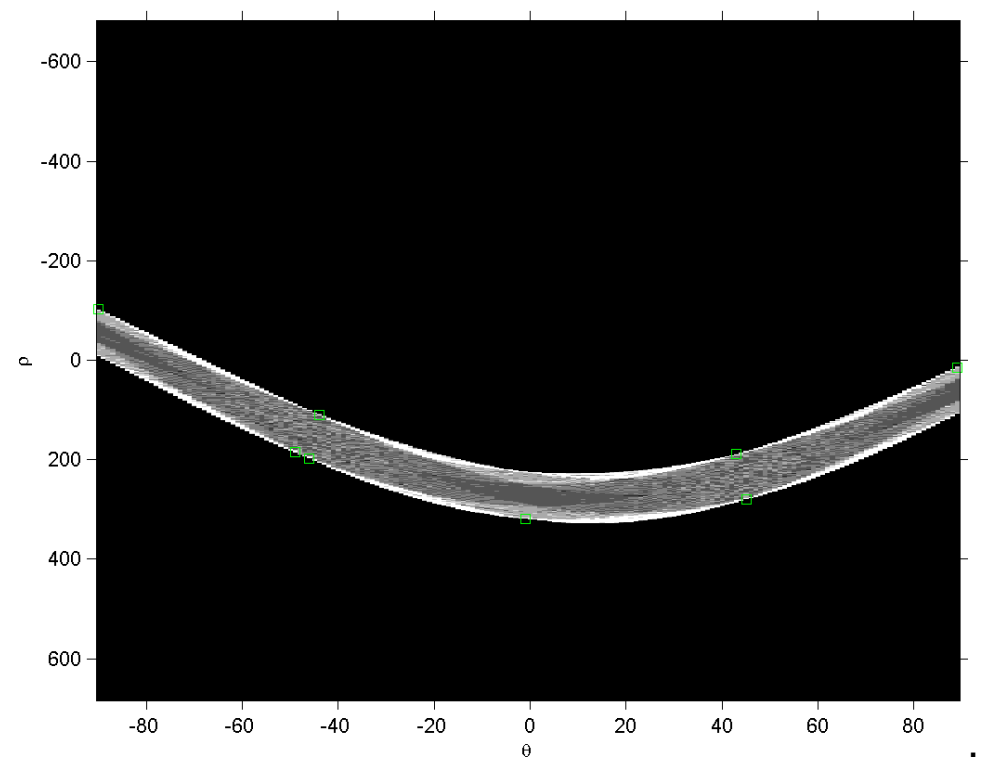

Figura 4. Transformada de Hough.

As retas encontradas são salvas em uma estrutura de dados que armazena as coordenadas dos pontos que determinam o início (em amarelo) e o final (em vermelho) de cada reta. A partir dessas informações, são exibidas na imagem original as retas detectadas que descrevem os limites da placa de pare na imagem original (Fig. 5).

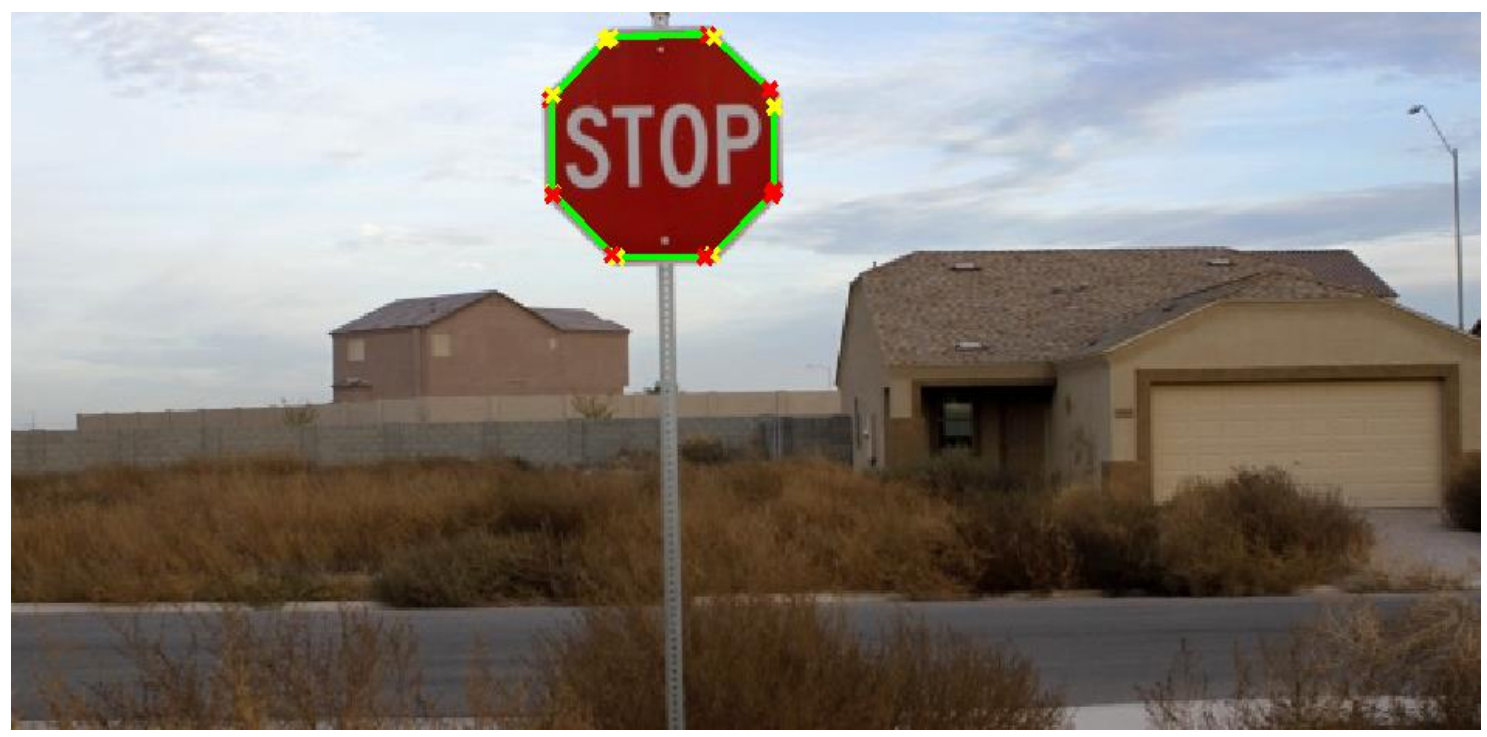

Figura 5. Linhas de borda reconhecidas. 
Para finalizar, é plotado um retângulo ao redor da placa observada, tendo-se como referência os pontos extremos que delimitam (acima, abaixo, à esquerda e à direita) a região provável de localização da placa na imagem de entrada, como pode ser observado na Fig. 6.

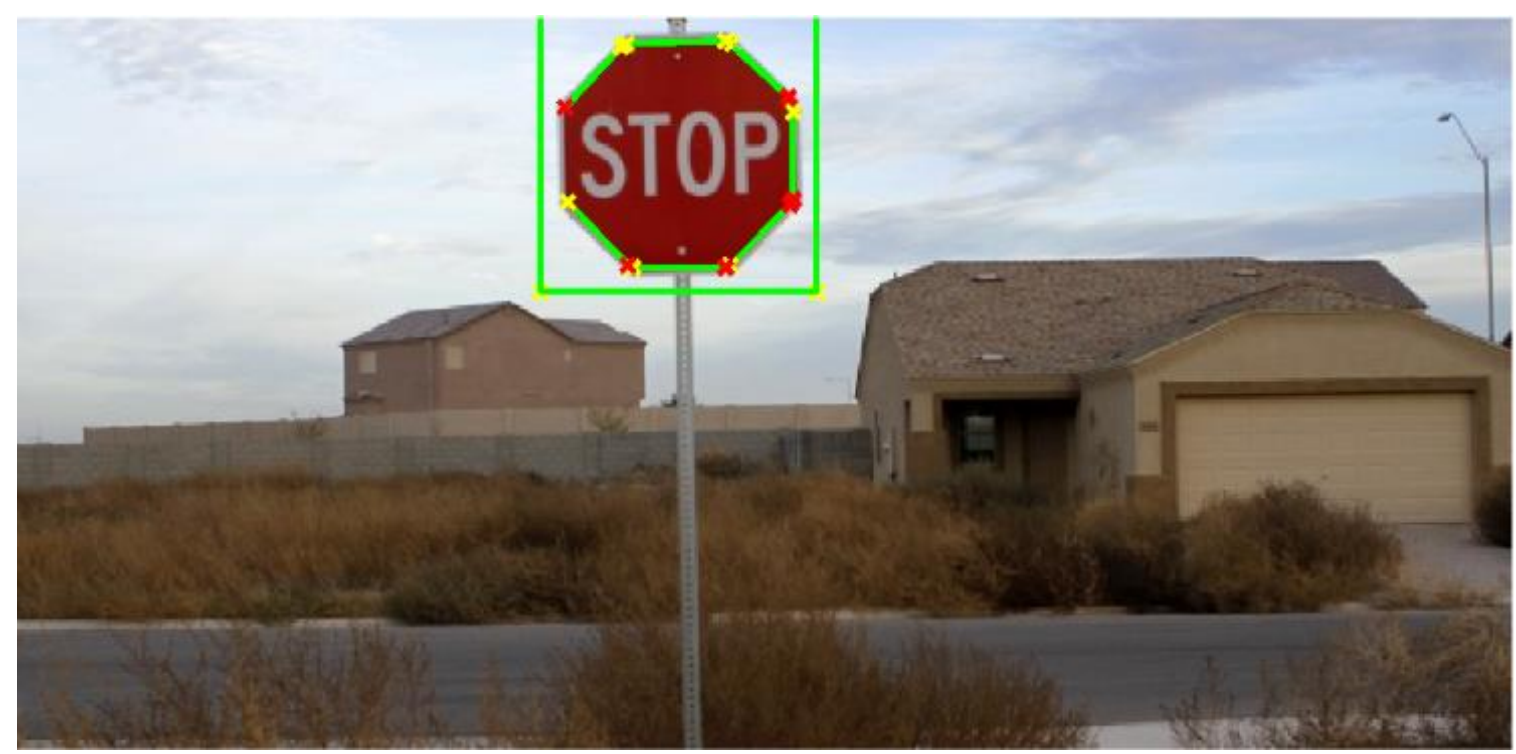

Figura 6. Resultado do algoritmo aplicado em uma imagem contendo uma placa de pare.

O resultado do procedimento descrito neste trabalho, pode então ser aplicado a algum método de reconhecimento de placas, como o apresentado em da Silva et al. (2012), com a vantagem de ter, juntamente com a região provável da placa, as coordenadas das retas que podem ser utilizadas para descrever a geometria do objeto detectado.

\section{EXPERIMENTOS}

Foram realizados experimentos utilizando uma base de dados disponível em BASILYAN (2016). Esta base de dados é composta por imagens com alguma placa de pare e imagens sem qualquer placa de pare. Para as imagens sem placas, foi detectado algum objeto em apenas quatro imagens, onde apresentava algum objeto com cor próxima ao vermelho. Nas imagens com alguma placa de pare, foi desenhado um retângulo contendo totalmente a placa em $70 \%$ dos casos.

A Fig. 7 apresenta alguns exemplos do resultado obtido pelo trabalho proposto na base de imagens utilizadas na etapa experimental. 


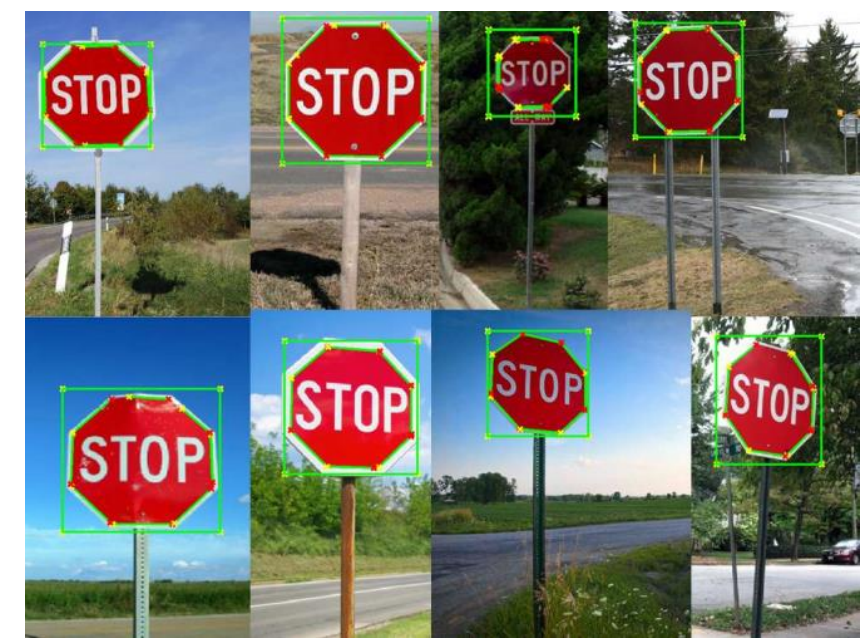

Figura 7. Resultado do algoritmo aplicado em imagens com placas de pare.

Levando-se em consideração o conjunto de imagens que continham alguma placa de pare, os resultados obtidos estão descritos na Tabela 1.

Tabela 1. Linhas de borda encontradas

\begin{tabular}{|c|c|}
\hline Conteúdo do retângulo & Quantidade \\
\hline Exatamente a placa & 46 \\
\hline Contém a placa & 5 \\
\hline Contém parte da placa & 1 \\
\hline Não contém a placa & 23 \\
\hline Total de imagens & 75 \\
\hline
\end{tabular}

Como se pode observar, em grande parte das imagens foram encontradas retas nas bordas da placa de pare, mas em algumas não foram encontradas. Esses falsos negativos ocorreram, em alguns casos, devido a oclusão parcial da placa por algum outro objeto presente na cena, imagem cortando parte da placa, imagens com a placa muito distante ou com baixa qualidade. Porém, em outros casos, casos houve apenas a falha do algoritmo.

\section{CONSIDERAÇÓES FINAIS}

O algoritmo desenvolvido detecta as coordenadas das retas nas bordas de objetos vermelhos em uma imagem de entrada e desenha um quadrado contendo estas linhas, possibilitando a detecção de uma possível placa de pare.

Considerando a base de dados utilizada, pôde-se observar que, em $70 \%$ dos casos, a metodologia proposta é capaz de identificar com precisão a área da imagem que contém a placa de parada obrigatória. Porém, vale destacar que, devido ao processo de 
segmentação de cores utilizado, e às restrições impostas neste processo referentes ao tamanho da placa em relação ao tamanho da imagem, possíveis variações cromáticas, ou a existência de objetos que possam ter características semelhantes ao objeto de interesse, o método pode falhar na detecção de placas em uma base de dados ampla e onde, por exemplo, estes tipos de restrições não são levados em consideração.

Foi possível constatar que a qualidade de algumas imagens do banco de imagens utilizado não contribuiu para um perfeito funcionamento da metodologia. Algumas imagens apresentaram a placa cortada ou muito distante, casos que o trabalho proposto ainda não é capaz de tratar adequadamente. Porém, conforme relatado, houve também alguns casos em que o algoritmo realmente falhou, mas acredita-se que isto possa ter ocorrido devido à variação cromática da placa na imagem Fig. 8. Como proposta de solução a este problema, pode-se incluir um processo de equalização cromática antes do processo de segmentação.

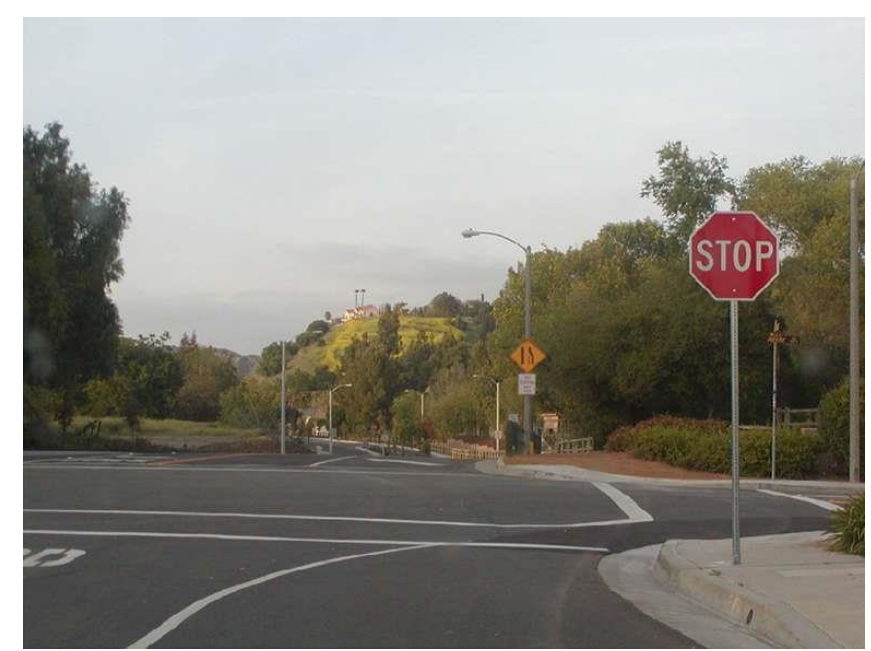

Figura 8. Exemplo de uma imagem em que a metodologia proposta não foi capaz de identificar a placa de parada obrigatória devido a baixa saturação da imagem de entrada.

O método apresentado pode ser modificado para encontrar outros tipos de placas de trânsito, como placas de regulamentação e placas de advertência. O objetivo deste método é ser utilizado em conjunto com outros métodos de reconhecimento de placas, como o algoritmo desenvolvido em da SILVA et al. (2012), que utiliza o descritor de características invariantes SIFT, ao fazer uma varredura inicial da imagem por completo e detectar uma possível placa de trânsito e fazendo uma primeira classificação para acelerar a identificação pelo SIFT. Essa combinação pode ser realizada em um possível trabalho futuro. 


\section{REFERÉNCIAS}

BASILYAN, M. (2016). Stop Sign Detection. Disponível em: <https://github.com/mbasilyan/Stop-Sign-Detection>. Acesso em 02 ago. 2017.

DA SILVA, F. A., ARTERO, A. O., DE PAIVA, M. S. V., AND BARBOSA, R. L. (2012). Uma metodologia para detectar e reconhecer placas de sinalização de trânsito. VIII Workshop de Visão Computacional.

GONZALEZ, R. AND WOODS, R. (2014). Processamento Digital De Imagens. Pearson Education, 3rd edition.

HOUGH, P. (1962). Method and means for recognizing complex patterns. U.S Patent 3069654 .

KIM, H. Y. (2017). Apostila: "Transformada de hough para detectar retas". http://www.lps.usp.br/hae/apostila/hough.pdf. Ultimo acesso em 14-08-2017.

LI, Q., LIANG, G., AND GONG, Y. (2015). A geometric framework for stop sign detection. In Signal and Information Processing (ChinaSIP), 2015 IEEE China Summit and International Conference on, pages 258-262. IEEE.

LOY, G. AND BARNES, N. (2004). Fast shape-based road sign detection for a driver assistance system. Intelligent Robots and Systems, 2004.(IROS 2004). Proceedings. 2004 IEEE/RSJ International Conference on, 1:70-75.

MATHWORKS (2017). Image processing toolbox. http://www.mathworks.com/help/images/. Ultimo acesso em: 10/07/2017.

SMITH, A. R. (1978). Color gamut transform pairs. ACM Siggraph Computer Graphics, 12(3):12-19.

ZHANG, S. (2016). Traffic sign detection for vision-based driver's assistance in land-based vehicles. Technical report, School of Aeronautics and Astronautics - Stanford University. 